\title{
Approximate Analytical Solution of Linear and Nonlinear Fractional Delay Differential Equations using Variational Iteration Method
}

\author{
Vineet Srivastava ${ }^{1 *}$ \\ ${ }^{1}$ Rajkiya Engineering College, Azamgarh, UP, India \\ *Corresponding author: Vineet Srivastava: vineeitbhu@gmail.com
}

\section{OPEN ACCESS}

Citation: Srivastava V. (2020) Approximate Analytical Solution of Linear and Nonlinear Fractional Delay Differential Equations using Variational Iteration Method. Open Science Journal 5(4)

Received: $15^{\text {th }}$ August 2020

Accepted: $9^{\text {th }}$ September 2020

Published: $14^{\text {th }}$ December 2020

Copyright: (c) 2020 This is an open access article under the terms of the Creative Commons Attribution License, which permits unrestricted use, distribution, and reproduction in any medium, provided the original author and source are credited.

Funding: The author(s) received no specific funding for this work

Competing Interests: The author has declared that no competing interests exists. Abstract:

In this paper, an approximate analytical method, New Variational Iteration Method (NVIM) is introduced in this paper for the approximate analytical solution of Fractional Delay Differential Equations (FDDEs). The algorithm is illustrated by studying initial value linear and nonlinear problems. The solutions thus obtained are presented and compare with exact solution. On the basis of comparison, it is concluding that only few iteration is required to get an approximate analytical solution.

Keywords: Fractional differential equation, Delay differential equation, Variational iteration method.

\section{Introduction}

Many complex problems of various scientific fields such as fluid dynamics, Bio-medical processes, solid state physics, plasma physics, mathematical biology, chemical kinetics etc. can be modeled by systems of linear or nonlinear ordinary differential equations (ODEs). In this row, due to further development, time delay systems have been modeled through Delay differential equations (DDEs). DDE is a differential equation in which time derivatives of dependent variables depends upon the solution and its derivatives at previous times. It has many applications in electronic, mechanical, chemical, transportation, biological systems, economic growth [1],[2],[3],[4],[5],,[6],[7],[8].

Fractional differential equations are another kind of differential equation which used for model the systems with long-range interactions or systems with memory [9], [10], [11]. If we combine above said two concepts i.e. Delay Differential Equations and Fractional Differential Equations, the real-world problems might be modelled more accurately and as result Fractional Delay Differential Equations (FDDEs) comes in the role. It has found that FDDEs have numerous applications in chemistry, physics, bioengineering, population 
dynamics, control systems, electro-chemistry and many other areas [12],[13], $[14],[15],[16]$. In this context, a number of papers are given which describes fractional Bloch equation with delay. Some recent references in this context see Refs. [17], [18] and the references therein. Interesting phenomenon such as chaos are observed in the form of fractional delay systems [19]. Hence, the fractional order delay differential equations have great importance for interdisciplinary area of research in recent years. Existence and uniqueness theorems on FDDEs have been discussed in [20], [21], [22] and [23].

The real-life mathematically modeled problems are still difficult to solve either theoretically or numerically. Most of the nonlinear fractional differential equations do not have exact analytic solutions, so approximation and numerical techniques have to be used. Many advanced methods such as Adomian Decomposition Method (ADM), Homotopy Perturbation Method (HPM), Homotopy Analysis Method (HAM), New Iterative Method (NIM) etc. have been developed to solve such problems and to get approximate analytical solution. The Variational Iteration Method (VIM) [24] is a relatively different approach to provide an analytical approximation to linear and nonlinear problems. They are particularly valuable as tools for applied mathematicians and scientist, because this method provide immediate and visible symbolic terms of approximate analytic solutions to both linear and nonlinear fractional differential equations without linearization or discretization in a very lucid manner. J. H. He $[24,25]$ was the first who proposed Variational Iteration Method and successfully applied to autonomous ordinary and partial differential equations and in other fields. In this row, Odibat and Momani [26] implemented the Variational Iteration Method to solve nonlinear ordinary differential equations of fractional order.

It is well known in the literature, this algorithm provides the solution in a rapidly convergent series obtained due to iterations [25, 26 \& 27]. The implementation of the variation iteration method in Ref. [28] amongst others has shown reliable results in that few terms only are needed to obtain accurate solutions.

The basic motivation of this work is the application of the variational iteration method (VIM) to solve the Linear and Nonlinear FDDEs. DaftardarGejji V. et al. [36] and Jhinga A. and Daftardar-Gejji V. [34, 37] have been already solved FDDEs numerically. But, the main objective of this paper is to extend the application of the variational iteration method as New Variational Iteration Method(NVIM) to provide approximate analytical solutions for initial value problems of linear and nonlinear FDDEs and to comparison with that exact solution.

\section{The method}

Consider the FDDE written in the form

$$
\begin{gathered}
L y(x)=f(x, y(x), y(g(x))), \quad 0 \leq x \leq 1, \\
y^{(i)}(0)=y_{0}^{i}, \quad i=0,1, \ldots \ldots, N-1, \\
y(x)=\Phi(x), \quad x \leq 0,
\end{gathered}
$$


where the differential operator $\mathrm{L}$ is given by

$$
L(\cdot)=\frac{d^{\alpha}(\cdot)}{d x^{\alpha}}, \quad m-1<\alpha \leq m,
$$

the variational iteration method was proposed by He [24], where a correction functional for Eq. (1) can be written as

$$
y_{n+1}(x)=y_{n}(x)+\int_{0}^{x} \lambda\left(L y_{n}(s)-f(s, \tilde{y}(s), \tilde{y}(g(s))) d s, \quad n \geq 0, \ldots\right.
$$

It is obvious that the successive approximations $y_{j}, j \geq 0$, can be established by determining $\lambda$ a general Lagrange multiplier, which can be identified optimally via the variational theory. The function $\tilde{y}_{n}$ is a restricted variation, which means $\delta \tilde{y}_{n}=0$. Therefore, we first determine the Lagrange multiplier $\lambda$ that will be identified optimally via integration by parts. The successive approximations $y_{n+1}(x), n \geq 0$ of the solution $y(x)$ will be readily obtained upon using the Lagrange multiplier obtained and by using selective function $y_{0}$. The initial values $y(0)$ and $y_{x}(0)$ are usually used for selecting the zeroth approximation $y_{0}$. With $\lambda$ determined, then several approximations $y_{j}(x), j \geq 0$, follow immediately. Consequently, the exact solution may be obtained by using

$$
y=\lim _{n \rightarrow \infty} y_{n}
$$

In what follows, we will apply the VIM method to five physical models given in the form of FDDE to illustrate the strength of the method and to establish exact solutions for these models.

\section{Application}

In this section, we discuss the some example on Fractional delay differential equation (FDDE) by using the variational iteration method as given in Section 2 .

Example $3.1[31,32] \quad$ Consider the Linear FDDE of first-order

$$
\frac{d^{\alpha} y(x)}{d x^{\alpha}}=\frac{1}{2} e^{x / 2} y\left(\frac{x}{2}\right)+\frac{1}{2} y(x), \quad 0 \leq x \leq 1, \quad y(0)=1, \quad 0<\alpha \leq 1
$$

which has the exact solution

$$
y(x)=e^{x}, \quad \text { for } \alpha=1 \text {. }
$$




$$
\begin{aligned}
& y_{n+1}(x)=y_{n}(x)+\int_{0}^{x} \lambda\left(\frac{d^{\alpha} y_{n}(s)}{d s^{\alpha}}-\frac{1}{2} e^{\frac{s}{2}} \tilde{y}_{n}\left(\frac{s}{2}\right)-\frac{1}{2} \tilde{y}_{n}(s)\right) d s, \\
& \delta y_{n+1}(x)=\delta y_{n}(x)+\delta \int_{0}^{x} \lambda\left(\frac{d^{\alpha} y_{n}(s)}{d s^{\alpha}}-\frac{1}{2} e^{\frac{s}{2}} \tilde{y}_{n}\left(\frac{s}{2}\right)-\frac{1}{2} \tilde{y}_{n}(s)\right) d s, \\
& \delta y_{n+1}(x)=(1+\lambda) \delta y_{n}(x)-\int_{0}^{x} \lambda^{\prime} \delta y_{n}(s) d s=0
\end{aligned}
$$

where $\tilde{y}_{n}$ is considered as restricted variations, which mean $\delta y_{n}=0$. Its stationary conditions can be obtained as follows

$$
1+\lambda(x)=0,\left.\quad \lambda^{\prime}(s)\right|_{s=x}=0 .
$$

The Lagrange multipliers, therefore, can be identified as $\lambda(x)=-1$

Therefore, from Eq.(6), we get

$$
y_{n+1}(x)=y_{n}(x)-\int_{0}^{x}\left(\frac{d^{\alpha} y_{n}(s)}{d s^{\alpha}}-\frac{1}{2} e^{\frac{s}{2}} y_{n}\left(\frac{s}{2}\right)-\frac{1}{2} y_{n}(s)\right) d s,
$$

Now, using initial condition, we get

$$
\begin{aligned}
y_{0}(x) & =1 \quad \text { and from Eq. (7) } \\
y_{1}(x) & =y_{0}(x)-\int_{0}^{x}\left(\frac{d^{\alpha} y_{0}(s)}{d s^{\alpha}}-\frac{1}{2} e^{\frac{s}{2}} y_{0}\left(\frac{s}{2}\right)-\frac{1}{2} y_{0}(s)\right) d s \\
& =1+\frac{1}{2}\left(2 e^{\frac{x}{2}}+x-2\right) \\
y_{2}(x) & =y_{1}(x)-\int_{0}^{x}\left(\frac{d^{\alpha} y_{1}(s)}{d s^{\alpha}}-\frac{1}{2} e^{\frac{s}{2}} y_{1}\left(\frac{s}{2}\right)-\frac{1}{2} y_{1}(s)\right) d s \\
& =1+\frac{1}{2}\left(2 e^{\frac{x}{2}}+x-2\right)-\frac{1}{8}\left(-\frac{16}{3} e^{\frac{3 x}{4}}+4 x-x^{2}-e^{\frac{x}{2}}(-4+2 x)\right)-\frac{1}{6}
\end{aligned}
$$

so on

when fourth iteration of the solution of this FDDE are compared with other methods [5, 6], we found that the present method is best as shown Table I. The differences between the exact and numerical solutions are given in Table I 
Table I. Comparison between exact solution \& solution obtain through VIM of Example 3.1

\begin{tabular}{|c|c|c|c|}
\hline $\mathrm{x}$ & Exact value & Present Method & Difference \\
\hline 0.2 & 1.2214 & 1.2214 & 0.0 \\
\hline 0.4 & 1.49182 & 1.49181 & 0.00001 \\
\hline 0.6 & 1.82212 & 1.82201 & 0.00011 \\
\hline 0.8 & 2.22554 & 2.22506 & 0.00048 \\
\hline 0.9 & 2.4596 & 2.45871 & 0.00089 \\
\hline
\end{tabular}

Example 3.2 [33] Consider the Linear FDDE of second-order

$$
\begin{aligned}
\frac{d^{\beta} y(x)}{d x^{\beta}} & =\frac{3}{4} y(x)+y\left(\frac{x}{2}\right)-x^{2}+2, \quad 0 \leq x \leq 1, \quad y(0)=0, \\
\frac{d y(0)}{d x} & =0, \quad \text { where } 1<\beta \leq 2
\end{aligned}
$$

To solve Eq. (8) by means of VIM, we construct a correction functional (see $(3))$,

$$
\begin{gathered}
y_{n+1}(x)=y_{n}(x)+\int_{0}^{x} \lambda\left(\frac{d^{2} y_{n}(s)}{d s^{2}}-\frac{3}{4} \tilde{y}_{n}(s)-\tilde{y}_{n}\left(\frac{s}{2}\right)+s^{2}-2\right) d s \\
\delta y_{n+1}(x)=\delta y_{n}(x)+\delta \int_{0}^{x} \lambda\left(\frac{d^{2} y_{n}(s)}{d s^{2}}-\frac{3}{4} \tilde{y}_{n}(s)-\tilde{y}_{n}\left(\frac{s}{2}\right)+s^{2}-2\right) d s \\
\delta y_{n+1}(x)=\left(1-\lambda^{\prime}\right) \delta y_{n}(x)+\lambda \delta y_{n}^{\prime}(x)+\int_{0}^{x} \lambda^{\prime \prime}(s) \delta y_{n}(s) d s=0,
\end{gathered}
$$

where $\tilde{y}_{n}$ is considered as restricted variations, which mean $\delta y_{n}=0$. Its stationary conditions can be obtained as follows

$$
\left(1-\lambda^{\prime}(x)\right)=0, \quad \lambda(x)=0,\left.\quad \lambda^{\prime \prime}(s)\right|_{s=x}=0
$$

The Lagrange multipliers, therefore, can be identified as

$$
\lambda(s)=(s-x)
$$

Therefore from Eq.(9), we get

$$
y_{n+1}(x)=y_{n}(x)+\int_{0}^{x}(s-x)\left(\frac{d^{\beta} y_{n}(s)}{d s^{\beta}}-\frac{3}{4} y_{n}(s)-y_{n}\left(\frac{s}{2}\right)+s^{2}-2\right) d s
$$

Now, using initial condition, we get

$$
\begin{aligned}
& y_{0}(x)=0, \text { and from Eq. }(10) \\
& \begin{array}{l}
y_{1}(x)=y_{0}+\int_{0}^{x}(s-x)\left(\frac{d^{\beta} y_{0}(s)}{d s^{\beta}}-\frac{3}{4} y_{0}(s)-y_{0}\left(\frac{s}{2}\right)+s^{2}-2\right) d s \\
=x^{2}-\frac{x^{4}}{12} .
\end{array}
\end{aligned}
$$




$$
\begin{aligned}
& y_{2}(x)=x^{2}-\frac{13 x^{6}}{480} \\
& y_{3}(x)=x^{2}-\frac{13 x^{8}}{114688} \\
& y_{4}(x)=x^{2}-\frac{2509 x^{10}}{2642411520} \\
& y_{5}(x)=x^{2}-\frac{1929421 x^{12}}{357169480335360} \\
& \ldots \ldots . . \\
& \text { ….... } \\
& \text { so on }
\end{aligned}
$$

If we take five iterations of the series, we get the difference between the exact and numerical solution given in Table II.

Table II. Comparison between exact solution \& solution obtain through VIM of Example 3.2

\begin{tabular}{|c|c|c|c|}
\hline $\mathrm{x}$ & Exact value & Present Method & Difference \\
\hline 0 & 0 & 0 & 0 \\
\hline 0.2 & 0.04 & 0.04 & $2.21265 \mathrm{E}-17$ \\
\hline 0.4 & 0.16 & 0.16 & $9.06301 \mathrm{E}-14$ \\
\hline 0.6 & 0.36 & 0.36 & $1.17589 \mathrm{E}-11$ \\
\hline 0.8 & 0.64 & 0.64 & $3.71221 \mathrm{E}-10$ \\
\hline 1.0 & 1.00 & 1.00 & $5.40198 \mathrm{E}-9$ \\
\hline
\end{tabular}

Example 3.3 Consider the Linear FDDE of third-order

$$
\begin{aligned}
& \frac{d^{\gamma} y(x)}{d x^{\gamma}}=-y(x)-y(x-0.3)+e^{-x+0.3}, \quad 0 \leq x \leq 1, \quad y(0)=1, \\
& \frac{d y(0)}{d x}=-1, \quad \frac{d^{2} y(0)}{d x^{2}}=1, \quad y(x)=e^{-x}, \quad x \leq 0, \text { where } 2<\gamma \leq 3
\end{aligned}
$$

To solve Eq. (11) by means of VIM, we construct a correction functional (see $(3))$,

$$
\begin{aligned}
& y_{n+1}(x)=y_{n}+\int_{0}^{x} \lambda\left(\frac{d^{3} y_{n}(s)}{d s^{3}}+\tilde{y}_{n}(s)+\tilde{y}_{n}(s-0.3)-e^{-s+0.3}\right) d s \\
& \delta y_{n+1}(x)=\delta y_{n}(x)+\delta \int_{0}^{x} \lambda\left(\frac{d^{3} y_{n}(s)}{d s^{3}}+\tilde{y}_{n}(s)+\tilde{y}_{n}(s-0.3)-e^{-s+0.3}\right) d s \\
& \delta y_{n+1}(x)=\left(1+\lambda^{\prime \prime}\right) \delta y_{n}(x)-\lambda^{\prime}(x) \delta y_{n}^{\prime}(x)+\lambda(x) \delta y_{n}^{\prime \prime}(x)-\int_{0}^{x} \lambda^{\prime \prime \prime}(x) \delta y_{n}(x) d s=0,
\end{aligned}
$$

where $\tilde{y}_{n}$ is considered as restricted variations, which mean $\delta y_{n}=0$. Its stationary conditions can be obtained as follows 


$$
\left.\lambda^{\prime \prime \prime}(s)\right|_{s=x}=0, \quad\left(1+\lambda^{\prime \prime}(x)\right)=0, \quad \lambda^{\prime}(x)=0,
$$

The Lagrange multipliers, therefore, can be identified as -

$$
\lambda(s)=-\frac{(s-x)^{2}}{2}
$$

Therefore from Eq.(12), we get

$$
y_{n+1}(x)=y_{n}(x)-\int_{0}^{x} \frac{(s-x)^{2}}{2}\left(\frac{d^{\gamma} y_{n}(s)}{d s^{\gamma}}+y_{n}(s)+y_{n}(s-0.3)-e^{-s+0.3}\right) d s
$$

Now, using initial condition, we get

$$
y_{0}(x)=c_{0}+c_{1} x+c_{2} x^{2}-e^{-x+0.3}
$$

where,

$c_{0}=2.3498588075759983, \quad c_{1}=-2.3498588075759983, \quad c_{2}=1.1749294037879991$.

using Eq.(14) in Eq.(13) we get the result for $\mathrm{n}=0,1,2,3, \ldots$.

In Table III, we make a comparison between the present method and the exact. When we take only three iterations from the series, we obtain the results given in Table III.

Table III. Comparison between exact solution \& solution obtain through VIM of Example 3.3

\begin{tabular}{|c|c|c|}
\hline $\mathrm{x}$ & Present Method & Exact \\
\hline 0.0 & 1.000000 & 1.000000 \\
\hline 0.2 & 0.818731 & 0.818731 \\
\hline 0.4 & 0.670320 & 0.670320 \\
\hline 0.6 & 0.548812 & 0.548812 \\
\hline 0.8 & 0.449330 & 0.449329 \\
\hline 1.0 & 0.367881 & 0.367879 \\
\hline
\end{tabular}

In the following examples, we apply the method to Non-Linear FDDE.

Example $3.4[34,35]$ Consider the Non-Linear FDDE of first-order

$$
\frac{d^{\alpha} y(x)}{d x^{\alpha}}=1-2 y^{2}\left(\frac{x}{2}\right), \quad 0 \leq x \leq 1, \quad y(0)=0, \quad 0<\alpha \leq 1
$$

To solve Eq. (11) by means of VIM, we construct a correction functional (see(3)),

$$
\begin{gathered}
y_{n+1}(x)=y_{n}(x)+\int_{0}^{x} \lambda\left(\frac{d^{\alpha} y_{n}(s)}{d s^{\alpha}}+2 \tilde{y}_{n}^{2}\left(\frac{s}{2}\right)-1\right) d s \\
\delta y_{n+1}(x)=\delta y_{n}(x)+\delta \int_{0}^{x} \lambda\left(\frac{d^{\alpha} y_{n}(s)}{d s^{\alpha}}+2 \tilde{y}_{n}^{2}\left(\frac{s}{2}\right)-1\right) d s \\
=(1+\lambda(x)) \delta y_{n}-\int_{0}^{x} \lambda^{\prime}(s) \delta y_{n}=0,
\end{gathered}
$$

where $\tilde{y}_{n}$ is considered as restricted variations, which mean $\delta y_{n}=0$. Its stationary conditions can be obtained as follows 


$$
(1+\lambda(x))=0,\left.\quad \quad \lambda^{\prime}(s)\right|_{s=x}=0,
$$

The Lagrange multipliers, therefore, can be identified as

$$
\lambda(x)=-1,
$$

Therefore from Eq.(15), we get

$$
y_{n+1}(x)=y_{n}(x)-\int_{0}^{x}\left(\frac{d^{\alpha} y_{n}(s)}{d s^{\alpha}}+2 y_{n}^{2}\left(\frac{s}{2}\right)-1\right) d s
$$

Now, using initial condition, we get

$$
\begin{aligned}
& y_{0}(x)=0, \quad \text { and from Eq. (16) } \\
& y_{1}(x)=y_{0}(x)-\int_{0}^{x}\left(\frac{d^{\alpha} y_{0}(s)}{d s^{\alpha}}+2 y_{0}^{2}\left(\frac{s}{2}\right)-1\right) d s \\
& y_{1}(x)=x, \\
& y_{2}(x)=x-\frac{x^{3}}{6}, \\
& y_{3}(x)=x-\frac{x^{3}}{6}+\frac{x^{5}}{120}-\frac{x^{7}}{8064},
\end{aligned}
$$

and using Taylor series, the exact solution $\mathrm{y}(\mathrm{x})=\sin \mathrm{x}$ is readily obtained.

Example 3.5 Consider the Non-Linear FDDE of third-order

$$
\frac{d^{\gamma} y(x)}{d x^{\gamma}}=-1+2 y^{2}\left(\frac{x}{2}\right), \quad 0 \leq x \leq 1, \quad y(0)=0, \quad \frac{d y(0)}{d x}=1, \quad \frac{d^{2} y(0)}{d x^{2}}=0,
$$

where $2<\gamma \leq 3$

$$
\begin{aligned}
y_{n+1}(x) & =y_{n}(x)+\int_{0}^{x} \lambda\left(\frac{d^{\gamma} y(s)}{d s^{\gamma}}-2 \tilde{y}^{2}\left(\frac{s}{2}\right)+1\right) d s \\
\delta y_{n+1}(x) & =\delta y_{n}(x)+\delta \int_{0}^{x} \lambda\left(\frac{d^{\gamma} y(s)}{d s^{\gamma}}-2 \tilde{y}^{2}\left(\frac{s}{2}\right)+1\right) d s \\
& =\left(1+\lambda^{\prime \prime}(x)\right) \delta y_{n}(x)+\lambda(x) \delta y_{n}^{\prime \prime}(x)-\lambda^{\prime}(x) \delta y_{n}^{\prime}(x)-\int_{0}^{x} \lambda^{\prime \prime \prime}(s) \delta y_{n}(s) d s=0,
\end{aligned}
$$

Its stationary conditions can be obtained as follows

$$
\left(1+\lambda^{\prime \prime}(x)\right)=0, \quad \lambda(x)=0, \quad \lambda^{\prime}(x)=0,\left.\quad \lambda^{\prime \prime \prime}(s)\right|_{s=x}=0,
$$

The Lagrange multipliers, therefore, can be identified as -

$$
\lambda(s)=-\frac{1}{2}(s-x)^{2}
$$

using this in Eq. (18) we get

$$
y_{n+1}(x)=y_{n}(x)-\frac{1}{2} \int_{0}^{x}(s-x)^{2}\left(\frac{d^{\gamma} y_{n}(s)}{d s^{\gamma}}-2 y_{n}^{2}\left(\frac{s}{2}\right)+1\right) d s
$$

Now, using initial condition, we get 


$$
\begin{aligned}
& y_{0}(x)=x \text { and from Eq. (19) } \\
& y_{1}(x)=y_{0}(x)-\frac{1}{2} \int_{0}^{x}(s-x)^{2}\left(\frac{d^{3} y_{0}(s)}{d s^{3}}-2 y_{0}^{2}\left(\frac{s}{2}\right)+1\right) d s \\
& =x-\frac{x^{3}}{6} \\
& y_{2}(x)=x-\frac{x^{3}}{6}+\frac{x^{5}}{120}-\frac{x^{7}}{5040}+\frac{x^{9}}{580608} \\
& \text { so on }
\end{aligned}
$$

for three iterations only, the comparison between the exact solution and approximate solution is shown in Table IV and it shows that the errors are very small.

Table IV. Comparison between exact solution \& solution obtain through VIM of Example 3.5

\begin{tabular}{|c|c|c|}
\hline $\mathrm{x}$ & Our Method & Exact solution \\
\hline 0.0 & 0.0 & 0.0 \\
\hline 0.2 & 0.19866933079506122 & 0.19866933079506122 \\
\hline 0.4 & 0.38941834230865047 & 0.3894183423086505 \\
\hline 0.6 & 0.5646424733950323 & 0.5646424733950355 \\
\hline 0.8 & 0.7173560908993959 & 0.7173560908995228 \\
\hline 1.0 & 0.841470984805614 & 0.84147109848078965 \\
\hline
\end{tabular}

From above examples, we can say that VIM gives approximate solutions in very good agreement with the exact solutions for only a few terms when solving DDEs. The programs for each case are written in the programming language Mathematica.

\section{Conclusion}

An advance technique, New Variational Iteration Method (NVIM), is used to solve the Fractional Delay Differential Equations (FDDEs) is presented. The method gives rapidly convergent successive approximations and handles linear and nonlinear problems in a similar manner. NVIM gives several successive approximations through using the iteration of the correction functional. In this method, there is no specific need to handle nonlinear terms as in Adomian Decomposition Method (ADM) [28]. All the numerical results of FDDEs obtained by using the NVIM described earlier show very good agreement with the exact solutions for only a few terms. We believe that the efficiency of the method gives it much wider applicability which been to be explored further. The author claims that it is first time to use NVIM to solve FDDEs. Author leaves more investigation for further research regarding general applicability of this VIM. 


\section{Acknowledgement}

The author is grateful to the referees for their valuable comments.

\section{References}

1.M.Jamshidi , C.M. Wang, A computational algorithm for large-scale nonlinear time-delays systems, IEEE Trans. Syst. Man Cybern. 14 (1984) 2-9 .

2.M.Lakshmanan , D.V. Senthilkumar , Dynamics of nonlinear time-delay systems, Springer Series Synergetics, 2010 .

3.G.Adomian , R. Rach , Nonlinear stochastic differential delay equations, J. Math. Anal. Appl. 91 (1983) 94-101 .

4.J.Baranowski, Legendre polynomial approximations of time delay systems, in: Proceeding of 12th International PhD Workshop, 1, 2010, pp. 15-20.

5.F.M.Asl , A.G. Ulsoy, Analysis of a system of linear delay differential equations, J. Dyn. Syst. Meas. Control 125 (2) (2003) 215-223 .

6.X.T. Wang, Numerical solution of delay systems containing inverse time by hybrid functions, Appl. Math. Comput. 173 (1) (2006) 535-546 .

7.Y.Takahashi, M.J. Rabins, D.M. Auslander, Control and dynamic systems, Addison Wesley, Massachusetts, 1970 .

8.J.K. Hale, Theory of functional differential equations, Springer-Verlag, New York, 1977 .

9.A.C. J. Luo and V. Afraimovich (Eds.), Long - range Interaction, Stochasticity and Fractional Dynamics, New York, Springer, 2010.

10.N.Laskin and G. M. Zaslavsky, Nonlinear fractional dynamics on a lattice with long -range interactions, Physica A 368, 38-4, 2006.

11.G.M.Zaslavsky, Hamiltonian Chaos and Fractional Dynamics, Oxford, Oxford University Press, 2005.

12.Daftardar-Gejji V (2014) Fractional calculus: theory and applications. Narosa Publishing House, New Delhi.

13.Epstein IR, LuoY(1991) Differential delay equations in chemical kinetics.Nonlinear models: the cross-shaped phase diagram and the oregonator. J Chem Phys 95(1):244-254

14.Davis L (2003) Modifications of the optimal velocity traffic model to include delay due to driver reaction time. Phys A Stat Mech Appl 319:557-567

15.Fridman E, Fridman L, Shustin E (2000) Steady modes in relay control systems with time delay and periodic disturbances. J DynSystMeas Control 122(4):732-737

16.Kuang Y (1993) Delay differential equations: with applications in population dynamics, vol 191. Academic Press, London

17.Bhalekar S, Daftardar-Gejji V, Baleanu D, Magin R (2011) Fractional Bloch equation with delay. Comput Math Appl 61(5):1355-1365

18.Baleanu D, Magin RL, Bhalekar S, Daftardar-Gejji V (2015) Chaos in the fractional order nonlinear Blochequation with delay. Commun Nonlinear Sci Numer Simul 25(1-3):41-49

19.Willé DR, Baker CT (1992) DELSOL - a numerical code for the solution of systems of delaydifferential equations. ApplNumer Math 9(3-5):223-234

20.Maraaba T, Baleanu D, Jarad F (2008a) Existence and uniqueness theorem for a class of delay differential equations with left and right Caputo fractional derivatives. J Math Phys 49(8):083507

21.Maraaba TA, JaradF,BaleanuD(2008b) On the existence and the uniqueness theorem for fractional differential equations with bounded delay within Caputo derivatives. Sci China Ser A Math 51(10):1775-1786

22.MorgadoML, Ford NJ, Lima PM(2013) Analysis and numerical methods for fractional differential equations with delay. J ComputAppl Math 252:159-168

23.Wang Z, Huang X, Shi G (2011) Analysis of nonlinear dynamics and chaos in a fractional order financial system with time delay. Comput Math Appl 62(3):1531-1539

24.J.H. He, Variational iteration method for delay differential equations, Commun. Nonlinear Sci. Numer. Simul. 2 (4) (1997) 235-236.

25.J.H. He, Variational iteration method-A kind of nonlinear analytical technique: Some examples, Internat. J. Non-Linear Mech. 34 (1999) 699-708.

26.Z. Odibat, S. Momani, Application of variational iteration method to nonlinear differential equations of fractional order, Int. J. Nonlinear Sci. Numer. Simul. 1 (7) (2006) 15-27. 
27.A. M. Wazwaz, The variational iteration method: A powerful scheme for handling linear and nonlinear diffusion equations, Int. J. Computers and Mathematics with Applications, 54 (2007), pp. 933-939.

28.A.M. Wazwaz, A comparison between the variational iteration method and Adomian decomposition method, Journal of Computational and Applied Mathematics, 207 (1) (2007) 129-136.

29.I. Podlubny, Fractional differential equations, Academic Press, San Diego, CA, 1999.

30.M. Caputo, Linear models of dissipation whose Q is almost frequency independent. Part II, J. Roy. Astr. Soc. 13 (1967) 529-539.

31.El-Safty, A., Salim, M. S. and El-Khatib, M. A. (2003). Convergent of the spline functions for delay dynamic system. Int. J. Comput. Math., 80(4), 509-518.

32.Shadia, M. (1992). Numerical solution of delay differential and neutral differential equations using spline methods. Ph. D Thesis, Assuit University.

33.El-Safty,A. (1993). Approximate solution of the delay differential equation $y=f(x, y(x), y(\alpha(x)))$ with cubic spline functions. Bull. Fac. Sci., Assuit Univ., 22(2-c), 67-73.

34.Ibrahim, M. A.-K., EI-Safty, A. and Abo-Hasha, S. M. (1995). 2h-step spline method for the solution of delay differential equations. Comput. Math. Appl., 29(8), 1-6.

35.EI-Safty, A. and Abo-Hasha, S. M. (1990). On the application of spline functions to initial value problem with retarded argument. Int. J. Comput. Math., 32, 137-179.

36.Daftardar-GejjiV, SukaleY, Bhalekar S (2015) Solving fractional delay differential equations: a newapproach. Fract Calc Appl Anal 18(2):400-418

37.Jhinga A. and Daftardar-Gejji V. (2019) A new numericalmethod for solving fractional delay differential equations, Computational and Applied Mathematics, 38(166) 